\title{
Gallbladder infection by trematodes
}

\author{
Claudio Tana $\cdot$ Andrea Mezzetti $\cdot$ Cosima Schiavone
}

Received: 2 April 2013/Accepted: 24 June 2013/Published online: 11 July 2013

(C) SIMI 2013

A 34-year-old-man presented to the emergency department (ED) because of the persistence of remittent fever (average temperature $39{ }^{\circ} \mathrm{C}$ ), malaise, jaundice and intense epigastrium pain radiating to the back, lasting 2 weeks. He reported a history of alcoholic cirrhosis complicated by ascites and encephalopathy. At home, the patient was treated regularly with aldosterone antagonists (spironolactone, $100 \mathrm{mg} /$ day) and loop diuretics (furosemide, $25 \mathrm{mg}$ twice daily). The patient reported having recently made a journey to China, during which he had eaten typical local food, such as the "Yusheng", a Chinese raw fish salad.

Blood pressure (BP), pulse and respiratory rates were 100/60 $\mathrm{mmHg}, 125$ beats/min and 16 breaths/min, respectively. The body temperature was $38.8^{\circ} \mathrm{C}$. The physical examination revealed right hypocondrium tenderness with local distension, positive Murphy's sign and reduction of peristalsis. The liver was palpable $3 \mathrm{~cm}$ below the right costal margin. Laboratory data showed the presence of hepatic dysfunction and cholestasis (AST $275 \mathrm{U} / \mathrm{L}$, ALT $56 \mathrm{U} / \mathrm{L}, \gamma$-GT $225 \mathrm{U} / \mathrm{L}$, ALP $203 \mathrm{U} / \mathrm{L}$, total and direct bilirubin 7.49 and $5.57 \mathrm{mg} / \mathrm{dL}$, respectively) and an increase in inflammatory markers (ESR $118 \mathrm{~mm} / \mathrm{h}$, leukocytosis with neutrophilia), altered coagulation blood tests

Electronic supplementary material The online version of this article (doi:10.1007/s11739-013-0974-2) contains supplementary material, which is available to authorized users.

C. Tana $\cdot$ A. Mezzetti $\cdot$ C. Schiavone $(\square)$

Department of Medicine and Science of Aging,

"G. d'Annunzio" University, Via dei Vestini 29,

66100 Chieti, Italy

e-mail: cschiavone@unich.it

C. Tana $\cdot$ C. Schiavone

Unit of Internistic Ultrasound, Department of Medicine and

Science of Aging, "G. d'Annunzio" University, Chieti, Italy
(PT $46 \%$, INR 1.7, aPTT 43 s) and mild thrombocytopenia $\left(109 \times 10^{3} / \mu \mathrm{L}\right)$. At abdominal ultrasound, the gallbladder showed an increased size (longitudinal and anteroposterior axis of $15 \times 6 \mathrm{~cm}$, respectively), thickened walls with a laminated appearance and lumen filled with echoic and stratified material, suggesting biliary sludge (Fig. 1a, b, arrows). Furthermore, in its dependent portion and in correspondence to the infundibulum, the gallbladder showed the presence of small, floating and non-shadowing echogenic foci that spontaneously changed in position and also in reaction to a light compression with the probe, taking on a flocculent appearance (Fig. 1a, b, arrowheads). The common bile duct (CBD) was increased in size (A-P axis of $6 \mathrm{~mm}$; Fig. 1b, asterisk). Because of the clinical suspicion of a parasitic infection corroborated by the history and ultrasound findings, a stool examination was performed, showing an infection by Clonorchis sinensis. The diagnosis was of acute cholecystitis and cholestatic jaundice by C. sinensis. The patient was successfully treated with antiparasite medications and showed a rapid clinical improvement, with early resolution of the fever, associated symptoms and laboratory findings. After 1 month, ultrasound showed a reduction of the gallbladder size, with atrophicsclerotic changes and death of the parasites. In view of the high risk of procedural complications (underlying chronic liver disease), surgery was contraindicated.

Endemic in Asian countries, parasitic infection of the biliary system can occur worldwide; indeed, isolated cases may occasionally be observed in travelers from nonendemic to endemic areas. The most frequently involved trematodes are Fasciola hepatica, C. sinensis and Opisthorchis viverrini. Among these, $C$. sinensis infection is the third most prevalent parasitosis, affecting 35 million people in the globe. It is more commonly encountered in countries such as Japan, China, Taiwan, Korea and Vietnam and it is 


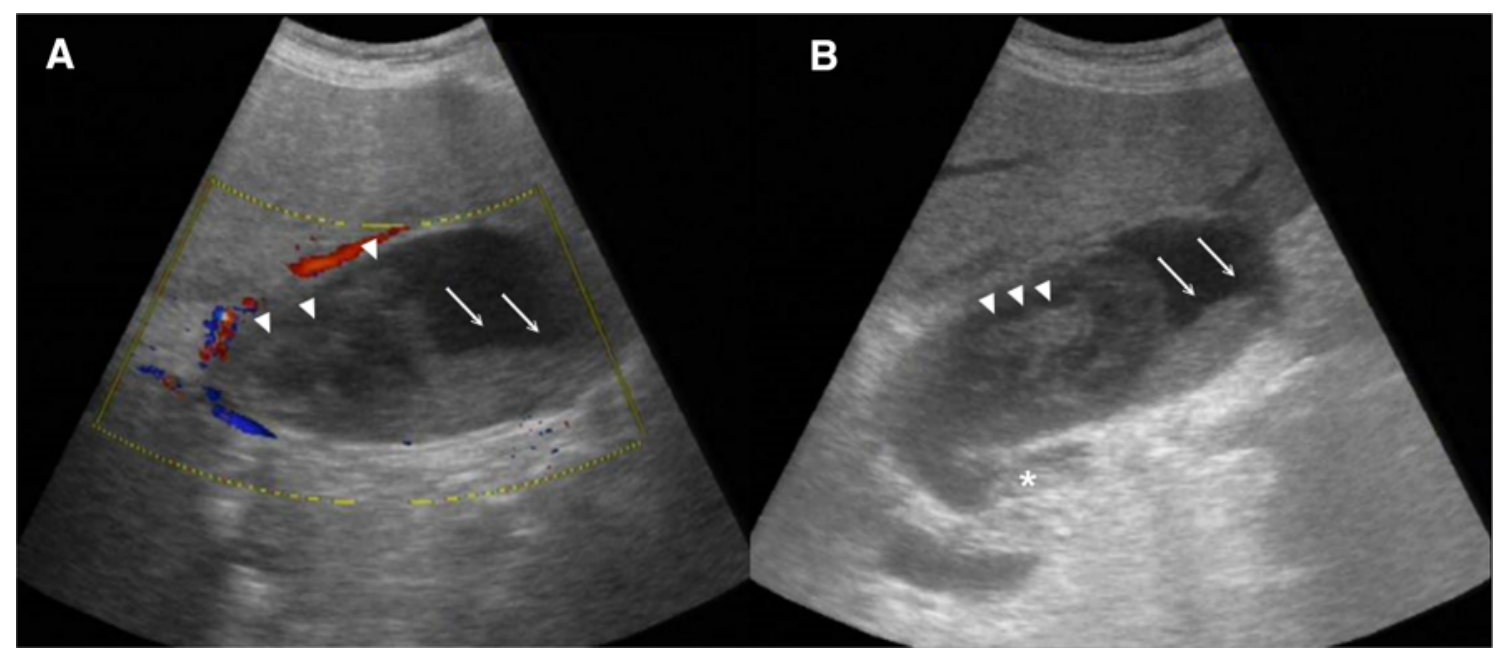

Fig. 1 Abdominal ultrasound showed biliary sludge (a and $\mathbf{b}$, arrows), non-shadowing floating echoes, compatible with flukes of Clonorchis sinensis (a and $\mathbf{b}$, arrowheads) and enlarged common bile duct $(6 \mathrm{~mm})$, secondary to the involvement by parasites (asterisk, b)

estimated that only in China about 15 million people are infected, constituting a serious public health problem [1-3]. After consumption of infected raw freshwater fish, the metacercariae exist in the duodenum and release the larvae; the latter, via the Vater's ampulla, can colonize the intrahepatic biliary system. Adult flukes of $C$. sinensis are usually small, ranging $8-15 \mathrm{~mm}$ and $1.5-4 \mathrm{~mm}$ (major and minor axis, respectively) and result in mild and non-specific symptoms and signs. Less frequently, they affect the common bile duct and gallbladder and can manifest with complications such as obstructive jaundice, cholangitis, periductal fibrosis, and cholangiocarcinoma [1, 3]. Thus, clinical findings are non-specific and a diagnosis can be made on the basis of the microscopic demonstration of eggs in the stool. The Kato-Katz technique is known as the most sensitive when compared to others, such as the formalin-ether technique and direct smear, but is difficult to obtain and can result in a false-negative diagnosis in cases of mild infection. Conversely, enzyme-linked immunosorbent assay (ELISA) can cross-react with other trematodes, resulting in a false-positive diagnosis, if other infections occur [2]. In this regard, ultrasound appears to be a valid diagnostic method, showing the best cost-effectiveness, when compared to other techniques (ready availability, low cost and non-invasiveness). Sonography can indeed show two specific findings in the case of active $C$. sinensis infection, such as the presence of increased periductal echogenicity (IPDE) (sensitivity $35 \%$, specificity $91 \%$; $p<0.001, R=0.11$ ) and floating echogenic foci in the gallbladder (FEFGB) (sensitivity $28 \%$, specificity $94 \%$; $p<0.001, R=0.09$ ). Others, such as diffuse dilatation of the intrahepatic bile ducts (DDIHD) and gallbladder distension, appear to have a lower diagnostic accuracy but can be useful as additional clues (sensitivity $67 \%$, specificity $48 \% ; p<0.01, R=0.03$; and sensitivity $3 \%$, specificity $100 \% ; p<0.05, R=0.02$, respectively) [4]. Furthermore, evident sonographic findings seem to have a linear and positive correlation with heavier infections, and their resolution may result in successful treatment (improvement of FEFGB, $p=0.004)$. In contrast, the persistence of floating echogenic foci in the gallbladder 1 year after the treatment may suggest a refractory active clonorchiasis [5]. Other examinations, such as computed tomography (CT) and magnetic resonance (MR) cholangiography, are indicated when sonography is not reliable and/or gives inconclusive results. They can show indirect signs, such as diffuse and uniform dilatation of the bile ducts, with significant thickening of the walls. The latter, associated with periductal enhancement on dynamic contrast-enhanced CT, seems to be useful to differentiate an active infection from cured clonorchiasis $[1,2]$.

In conclusion, ultrasound can be useful for the initial assessment of a suspected gallbladder infection by $C$. sinensis. By demonstrating the presence of spontaneous floating of non-shadowing echogenic foci in the gallbladder, sonography can exhibit a good diagnostic value, especially in the presence of suggestive history. However, because of its low sensitivity, a negative ultrasound does not exclude the presence of flukes, and a correct diagnosis can be made after identification of eggs in the stool. A proper diagnosis is essential, as it indicates an appropriate drug treatment, thus avoiding an unnecessary (at least initially) surgical approach. The latter can be reserved to patients with resistance to antiparasitic therapy [2].

\section{Conflict of interest None.}




\section{References}

1. Lim JH, Kim SY, Park CM (2007) Parasitic diseases of the biliary tract. AJR Am J Roentgenol 188(6):1596-1603

2. Choi D, Hong ST (2007) Imaging diagnosis of clonorchiasis. Korean J Parasitol 45(2):77-85

3. Lun ZR, Gasser RB, Lai DH et al (2005) Clonorchiasis: a key foodborne zoonosis in China. Lancet Infect Dis 5(1):31-41
4. Choi D, Hong ST, Lim JH et al (2004) Sonographic findings of active Clonorchis sinensis infection. J Clin Ultrasound 32(1): $17-23$

5. Choi D, Jeon YH, Lee GC et al (2009) Changes in sonographic findings after treatment of patients with clonorchiasis in a heavy endemic area. Korean J Parasitol 47(1):19-23 